\title{
Práticas inovadoras em saúde coletiva: ferramenta re-leitora do processo saúde-doença*
}

\author{
INNOVATOR PRACTICES IN COLLECTIVE HEALTH: RE-READING TOOL OF \\ THE HEALTH DISEASE PROCESS
}

\section{PRÁCTICAS INNOVADORAS EN SALUD COLECTIVA: HERRAMIENTA RE-LECTORA DEL PROCESO SALUD ENFERMEDAD}

\section{Marcia Regina Cubas', Emiko Yoshikawa Egry²}

\section{RESUMO}

O objetivo deste artigo foi apresentar as bases para a construção de uma ferramenta re-leitora do Processo Saúde-doença, por meio da relação dialógica entre categorias sociais e as variáveis funcionais, contidas no instrumento CIPESC ${ }^{\circledR}$ de Curitiba. Ancorou-se nos marcos conceituais apresentados na Teoria da Intervenção Práxica da Enfermagem em Saúde Coletiva. Aferramenta cons-tituiu-se de um modelo analítico de apoio à tomada de decisão, pretendendo o desenvolvimento de data warehouse. Os dados do prontuário eletrônico do paciente, relacionados às categorias: $\mathrm{Ne}-$ cessidade, Classe Social, Gênero, Etnia e Geração, desvelam a face social e atribuem vulnerabilidade aos grupos sociais homogêneos. Foram visualizados quatro graus de vulnerabilidade, qualificados por 11 marcadores. $\mathrm{O}$ estudo re-meteu à reflexão de que as desigualdades reproduzem-se ao longo do tempo e transformam grupos em alvos de imobilidade social. Embora existam lacunas para visualização das categorias sociais, o instrumento permitiu visualizar a face coletiva de famílias e grupos sociais.

\section{DESCRITORES}

Vulnerabilidade em saúde.

Processo saúde-doença.

Enfermagem.

Classificação.

Determinação de necessidades de cuidados de saúde.

\begin{abstract}
This study aims to present the basis to build a re-reading tool of the Health-Disease Process which relies on dialogical relation among social categories and the functional variables found in the a consultation instrument. The tool is an analytical model for decision support that intends the development of data warehousing. Data from the electronic records of nursing consultation were related to the categories: need, social class, gender, race and generation, unveil the social aspects of the health - disease process and assign degrees of vulnerability to homogeneous social groups. Four degrees of vulnerability can be observed that are qualified by eleven markers. The tool lead to the reflection that inequalities are reproduced as time goes by and transforms groups in targets from the social immobility. Although there are some gaps for the visualization of the social categories, the tool enables to visualize the collective face of families and social groups.
\end{abstract}

\section{KEY WORDS}

Health vulnerability.

Health-disease process.

Nursing.

Classification.

Needs assessment.

\section{RESUMEN}

El objetivo de este artículo fue presentar las bases para la construcción de una herramienta relectora del proceso salud-enfermedad, por medio de una relación entre las categorías sociales y las variables funcionales de un instrumento utilizado en las consultas de enfermería. La herramienta se constituyó en un modelo analítico de apoyo para tomar decisiones, pretendiendo el desarrollo de data warehouse. Las informaciones fueron obtenidas de las bases electrónicas de los pacientes, en relación a las categorías: necesidad, clase social, género, etnia y generación. Esas categorías revelaron el aspecto social y atribuyeron vulnerabilidad a los grupos sociales homogéneos. Los resultados llevan a la reflexión que las desigualdades se reproducen a lo largo del tiempo y transforman grupos en albos de inmovilidad social. A pesar de existir lagunas para visualizar las categorías sociales, el instrumento permitió visualizar el aspecto colectivo de familias y grupos sociales.

\author{
Extraído da tese \\ "CIPESC ${ }^{\circledR}$ Curitiba: \\ proposta de uma \\ ferramenta re-leitora \\ da face coletiva do \\ processo saúde \\ doença", Escola de \\ Enfermagem,Universi- \\ dade de São Paulo, \\ 2006. \\ 1 Enfermeira. Doutora \\ em enfermagem. \\ Professora Assis- \\ tente do Programa de \\ Pós-graduação em \\ Tecnologia em Saúde \\ da Pontifícia Univer- \\ sidade Católica do \\ Paraná (PUCPR). \\ Curitiba, PR, Brasil. \\ m.cubas@pucpr.br \\ 2 Enfermeira. Profes- \\ sora Titular do Depar- \\ tamento de Saúde \\ Coletiva da Escola \\ de Enfermagem, \\ Universidade de São \\ Paulo (EEUSP). \\ Pesquisadora do \\ CNPq. São Paulo, \\ SP, Brasil.
}

\section{DESCRIPTORES}

Vulnerabilidad en salud.

Proceso salud-enfermedad.

Enfermería.

Clasificación.

Evaluación de necesidades. 


\section{INTRODUÇÃO}

Entre 1996 e 2000, a Associação Brasileira de Enfermagem - ABEn - desenvolveu o projeto Classificação Internacional das Práticas de Enfermagem em Saúde Coletiva CIPESC, contribuição brasileira à Classificação Internacional de Práticas de Enfermagem - CIPE ${ }^{\circledR(1)}$. O desenvolvimento do projeto teve abrangência nacional, superando as diversidades socioeconômicas, culturais e de acesso geográfico.

Dentre os resultados apresentados, discutiu-se que, muito embora os avanços conquistados pelo trabalho da Enfermagem no âmbito do Sistema Único de Saúde - SUS, no que se refere ao modelo de atenção à saúde, o trabalho coletivo ainda é tomado por uma somatória de ações particularizadas $^{(2)}$. Não menos importante que a análise do processo de trabalho da Enfermagem brasileira, o CIPESC produziu uma contribuição aos fenômenos de Enfermagem da CIPE $^{\circledR}$, incorporando termos empregados no Brasil e lançando a marca CIPESC ${ }^{\circledR}$, um instrumento para a prática profissional da enfermeira na saúde coletiva ${ }^{(3)}$.

Ao pretender classificar suas práticas no espaço da saúde coletiva, a enfermeira depara-se com aspetos subjetivos do Processo Saúde-doença, que impulsiona mudanças significativas na sua prática profissional. O indivíduo assistido passa a ser visto como um sujeito portador de uma história de vida, determinada pela forma de organização da sociedade em que vive e pela sua inserção em um grupo social recortado por classe social, etnia, geração e relações de gênero.

Autores $^{(4)}$ afirmam que a CIPESC possibilita visibilizar as estruturas maiores que organizam o trabalho da Enfermagem, ao mesmo tempo em que pode se tornar um instrumento poderoso, potencializador da avaliação processual dos resultados, benefícios e impactos da ação da Enfermagem.

Curitiba foi a primeira cidade do Brasil a implantar a CIPESC $^{\circledR}$ num sistema operacional informatizado ${ }^{(5)}$. Para isso, precisou enfrentar as contradições entre a saúde coletiva que vê o indivíduo na sua totalidade, dinamicidade e historicidade, portanto na sua face coletiva - e a prática assistencial da saúde pública que recorta o indivíduo pelo seu ciclo vital, patologia ou sexo e que percebe o social como externo ao sujeito.

Este artigo apresenta os resultados de um estudo realizado em Curitiba, em resposta à seguinte questão orientadora: existe potencialidade nos instrumentos de trabalho da Enfermagem em saúde coletiva para capturar a face coletiva dos indivíduos? Teve por objetivo formular as bases para a construção de uma ferramenta re-leitora do processo saúde-doença, privilegiando a face coletiva, por meio da relação dialógica entre categorias sociais e as variáveis funcionais contidas no instrumento CIPESC $^{\circledR}$ - Curitiba.

\section{MÉTODO}

A presente investigação ancora-se nos marcos conceituais da Saúde Coletiva, apresentados na Teoria da Intervenção Práxica da Enfermagem em Saúde Coletiva TIPESC $^{(6)}$. Seus momentos compreendem a: captação da realidade objetiva (RO); a interpretação da RO; construção do projeto de intervenção na RO; a intervenção na $\mathrm{RO}$ e reinterpretação da RO. O desenvolvimento da TIPESC é feito em etapas de forma sistematizada, porém não perde de vista o caráter de totalidade e de interprenetrabilidade. Cada etapa revela um momento hegemônico de abordagem, quando se destaca uma fase em relação às demais, no entanto podese verificar que todas acontecem simultaneamente em um determinado tempo ${ }^{(6)}$.

A base empírica deste estudo foi constituída por: sistema informatizado utilizado pela enfermagem na Secretaria Municipal da Saúde de Curitiba, relatórios de produção de consultas de enfermagem de agosto de 2004 a julho de 2006, resultados da captação da RO decorrente do subprojeto Classificação das Práticas de Enfermagem em Saúde Coletiva - CIPESC ${ }^{\circledR}$ : captação da realidade objetiva do cenário de Curitiba, Paraná ${ }^{(7-8)}$; material produzido no seminário com o grupo de consultores e resultado da aplicação da Matriz de Impactos Cruzados.

O universo de sujeitos da pesquisa foi composto por: grupo de coordenação da Sistematização das Práticas de Enfermagem em Saúde Coletiva da Secretaria Municipal de Saúde (SMS) de Curitiba; grupo de construção dos elementos da prática de enfermagem da SMS; consultoras Professoras Doutoras do Departamento de Saúde Coletiva da Escola de Enfermagem da Universidade de São Paulo (EEUSP) e doutorandas da Universidade Federal do Paraná e Pontifícia Universidade Católica do Paraná.

O estudo desenvolveu-se por fases, a saber: re-interpretação da RO por meio dos resultados do Projeto Unificado: As Transformações dos Processos de Trabalho da Enfermagem em Saúde Coletiva: instrumentalizando para uma prática inovadora ${ }^{(9)}$; relação das variáveis funcionais contidas no prontuário informatizado da SMS, em banco de dados do Instituto de Pesquisa e Planejamento Urbano de Curitiba IPPUC; análise das co-relações possíveis entre as variáveis e a construção de subcategorias que estabelecem relação dialógica com as categorias interpretativas; formatação da arquitetura dos dados operacionais; organização das co relações entre as variáveis, estabelecendo impactos entre 
as mesmas para estabelecer graus de vulnerabilidade por meio de uma Matriz de Impactos Cruzados ${ }^{(10)}$.

Os preceitos éticos foram resguardados pela apreciação do Projeto pelo Comitê de Ética da Escola de Enfermagem da Universidade de São Paulo (Processo n. 415/2004), assim como foi examinado pelo Comitê de Ética em Pesquisa da SMS, dando cumprimento à Resolução 196/96 do Conselho Nacional de Saúde.

\section{RESULTADOS E DISCUSSÃO}

A ferramenta re-leitora constitui um modelo analítico de apoio à tomada de decisão, ou seja, ao realizar um atendimento a um indivíduo, o sistema poderá oferecer à enfermeira uma síntese de sua inserção no coletivo por meio de uma estruturação de dados que são alimentados na base operacional. Para isso, buscou-se ancoragem no Sistema de Apoio à Decisão - SAD, pretendendo o desenvolvimento da data warehouse $^{(11)}$, o qual permite que dados departamentalizados de sistemas operacionais possam ser integrados em uma única fonte.

Ao iniciar este desenvolvimento, realizou-se um inventário dos dados disponíveis no prontuário eletrônico do paciente, os quais poderiam ser relacionados às categorias interpretativas propostas: necessidade, classe social, gênero, etnia e geração. O prontuário eletrônico do paciente permite levantar características do espaço singular e, ao captálas, relacionando-as às demais categorias, desvelar a face social das necessidades de saúde, conseqüentemente, atribuir vulnerabilidade aos grupos sociais homogêneos, que pode ser gradativa, instável ao longo do tempo e multidimensional, podendo apresentar-se vulnerável sob certo aspecto e protegida em outro ${ }^{(12)}$.

Em relação à primeira categoria interpretativa - Necessidade, os dados disponíveis no prontuário estão configurados de forma a organizar os diagnósticos e intervenções de Enfermagem dentro do referencial teórico do Processo de Enfermagem Wanda Horta ${ }^{(13)}$, que se baseia na teoria das necessidades humanas de Maslow. Desta forma, para captála, o modelo da ferramenta interliga as variáveis oriundas dos diagnósticos e intervenções de Enfermagem que compõe a sub-categoria perfil saúde-doença. Para compor a primeira articulação, os elementos constituintes da nomenclatura de diagnósticos foram classificados em: diagnósticos destrutivos (riscos e contra valores) e diagnósticos protetores (potencialidades e valores), que explicitam o perfil de saúde-doença no espaço singular. As intervenções de Enfermagem estão dispostas no prontuário, conforme a classificação de verbos de ação da CIPESC $^{\circledR}$, ou seja: Observar, Gerenciar, Informar, Desempenhar e Atender/Cuidar ${ }^{(3)}$. Para compor este modelo, as intervenções de Enfermagem listadas foram divididas em dois grupos: as que superam processos destrutivos e as que potencializam processos protetores.
Em relação à segunda categoria: Classe Social, o prontuário eletrônico não permite a explicitação de todas as variáveis utilizadas para categorizar classes, conforme a inserção no sistema produtivo. Esta é uma categoria que explicita os diferentes modos de vida e é capaz de determinar o perfil epidemiológico do espaço particular. Para formulação do modelo de ferramenta, agregaram-se variáveis que compõem as subcategorias capazes de organizar frações de classes sociais, ou seja, a micro-área, a condição do domicílio, a inserção no mercado de trabalho, a renda e a escolaridade.

A categoria Gênero está relacionada a desigualdades, conectada intimamente a outras categorias sociais e ligadas aos três níveis de formulação: a estrutura social, a implementação de políticas públicas e a assistência (cuidado) ${ }^{(14)}$. O prontuário eletrônico oferece apenas a sub-categoria sexo, mas a ferramenta deverá permitir a linkagem com outras sub-categorias para que a categoria Gênero seja desvelada.

A categoria Etnia, como identidade de povos, não é explicitada no prontuário eletrônico, nem mesmo por sua possível relação com a variável raça. Neste ponto, para a ferramenta, pode-se recorrer apenas aos dados de raça provenientes do IPPUC - Instituto de Pesquisa e Planejamento Urbano de Curitiba. É uma categoria que, articulada a gênero e classe, desvela a formação dos diferentes grupos sociais.

A Geração, entendida como processo de construção da responsabilidade civil, faz-se presente apenas como divisão de faixa etária, e não na sua dinamicidade com a cultura e o modo de vida que determina a construção de identidade nas diferentes faixas etárias. No entanto, esta categoria permite avaliar vulnerabilidades de grupos que se encontram em pólos que necessitam de maior proteção: os idosos, as crianças e os adolescentes.

Para estabelecer os diferentes graus de vulnerabilidade dos grupos homogêneos, utilizou-se a Matriz de Impactos Cruzados, cuja finalidade é hierarquizar as variáveis em termos de motricidade ${ }^{(a)}$, dependência e associação ${ }^{(10)}$. Primeiramente, essa matriz foi aplicada a consultoras e a enfermeiras da Secretaria Municipal de Saúde de Curitiba. As escalas decorrentes das matrizes individuais foram organizadas de forma a compor uma matriz única que expôs algumas situações em relação às categorias interpretativas.

Os resultados permitem inferir que as variáveis que possuem maior influência no sistema são: estar sem emprego; morar em área de risco e não ter renda. As condições com maior dependência de outras são: morar em área de risco;

(a) A matriz de impactos cruzados permite analisar a influência de uma variável sobre as outras. São categorias de três formas: as variáveis dependentes, as quais possuem uma dependência direta das outras; as variáveis motrizes, as quais possuem capacidade de determinar outras variáveis e as variáveis de ligação, que apresentam simultaneamente alta dependência e motricidade, ou seja, determinam e são determinadas por outras variáveis. 
não ter renda; ter renda de 1 a 3 salários mínimos; não ter esgoto em casa; jogar dejetos a céu aberto; não ter instrução ou ter menos de 1 ano de estudo.

A subcategoria sexo não apresentou diferenças, nem na motricidade, tampouco na dependência. Na subcategoria faixa etária, os dados revelam que todas as condições possuem dependência maior que a motricidade, porém a condição de ser adolescente mostra um diferencial na dependên- cia que não deve ser descartado. Foi na subcategoria raça que ocorreu a maior diferenciação: tanto na motricidade, quanto na dependência.

Esses resultados, somados à captação da realidade objetiva curitibana e aos relatórios de discussão com o grupo consultor, constituíram quatro divisões de agregação dos dados, composta por 11 marcadores, que estabeleceram diferentes graus de vulnerabilidade (Quadro 1).

Quadro 1 - Condições que compõem os diferentes graus de vulnerabilidade de grupos homogêneos - Curitiba - 2006

\begin{tabular}{|c|c|c|c|}
\hline $\begin{array}{c}\text { Vulnerabilidade } \\
\text { Zero }\end{array}$ & Vulnerabilidade Baixa & Vulnerabilidade Média & Vulnerabilidade Alta \\
\hline Moradores de área não risco & Morador em área não risco & Morador em área de risco & Morar em área de risco \\
\hline Água tratada & Água sem tratamento & Água sem tratamento & Água sem tratamento \\
\hline $\begin{array}{l}\text { Abastecimento de água em rede } \\
\text { ou poço (tratada) }\end{array}$ & $\begin{array}{l}\text { Abastecimento de poço não } \\
\text { tratado }\end{array}$ & $\begin{array}{l}\text { Abastecimento de poço não } \\
\text { tratado }\end{array}$ & $\begin{array}{l}\text { Abastecimento de poço não } \\
\text { tratado }\end{array}$ \\
\hline Habitação de tijolo ou madeira & Habitação de tijolo ou madeira & Habitação de tijolo ou madeira & Habitação material aproveitado \\
\hline Com energia elétrica & Com energia elétrica & Sem energia elétrica & Sem energia elétrica \\
\hline $\begin{array}{l}\text { Destino dos dejetos em esgoto ou } \\
\text { fossa }\end{array}$ & $\begin{array}{l}\text { Destino dos dejetos em esgoto ou } \\
\text { fossa }\end{array}$ & Destino dos dejetos a céu aberto & Destino dos dejetos a céu aberto \\
\hline Lixo coletado & Lixo queimado ou a céu aberto & Lixo queimado ou a céu aberto & Lixo queimado ou a céu aberto \\
\hline $\begin{array}{l}\text { Número de peças do domicílio } \\
\text { acima de } 5 \text { cômodos }\end{array}$ & $\begin{array}{l}\text { Número de peças de } 3 \text { a } 5 \\
\text { cômodos }\end{array}$ & $\begin{array}{l}\text { Número de peças de } 3 \text { a } 5 \\
\text { cômodos }\end{array}$ & $\begin{array}{l}\text { Número de peças de } 1 \text { a } 3 \\
\text { cômodos }\end{array}$ \\
\hline $\begin{array}{l}\text { Trabalhador formal ou } \\
\text { aposentado }\end{array}$ & $\begin{array}{l}\text { Trabalhador formal ou } \\
\text { aposentado }\end{array}$ & $\begin{array}{l}\text { Trabalhador informal, aposentado } \\
\text { ou desempregado }\end{array}$ & $\begin{array}{l}\text { Trabalhador informal ou } \\
\text { desempregado }\end{array}$ \\
\hline $\begin{array}{l}\text { Escolaridade superior a } 10 \text { anos } \\
\text { de estudo }\end{array}$ & $\begin{array}{l}\text { Escolaridade de } 8 \text { a } 10 \text { anos de } \\
\text { estudo }\end{array}$ & $\begin{array}{l}\text { Escolaridade de } 4 \text { a } 7 \text { anos de } \\
\text { estudo }\end{array}$ & $\begin{array}{l}\text { Escolaridade de } 1 \text { a } 3 \text { anos de } \\
\text { estudo ou sem instrução }\end{array}$ \\
\hline $\begin{array}{l}\text { Renda maior que } 11 \text { salários } \\
\text { mínimos }\end{array}$ & Renda de 8 a 10 salários mínimos & Renda de 4 a 7 salários mínimos & $\begin{array}{l}\text { Não ter renda ou renda de } 1 \text { a } 3 \\
\text { salários mínimos }\end{array}$ \\
\hline
\end{tabular}

Diante deste quadro, foi necessário estabelecer alguns parâmetros para extração dos dados: a condição de morar em área de risco, água sem tratamento e lixo não coletado, se encontradas isoladamente, configuram algum grau de vulnerabilidade; as condições de morar em área de risco, estar sem emprego e não ter renda, se encontradas em conjunto, configuram automaticamente um grau de vulnerabilidade alta; a condição de ser adolescente ou ser negro, em conjunto com as outras condições referentes a algum grau de vulnerabilidade, fazem com que o indivíduo seja alocado no maior grau de vulnerabilidade e os relatórios da subcategoria perfil saúde-doença devem ser retirados de acordo com o grau de vulnerabilidade que os determinados grupos homogêneos se situem.

\section{CONCLUSÃO}

O Plano Municipal de Saúde de Curitiba 2002-2005 ${ }^{(15)}$ pautou a política de saúde num modelo de assistência que propiciava uma prática solidária, com melhora do acesso e da resolubilidade, reafirmando os princípios de cidadania. A contribuição da Enfermagem ao Plano Municipal, faz-se presente ao referir que seu trabalho defende a política do cuidar

comprometido com a busca de soluções para os problemas vividos pelas pessoas/comunidades que precisam dos serviços de saúde ${ }^{(15)}$.

Desta forma, ao compreender o trabalho da Enfermagem como parcela do trabalho em saúde, presente na produção dos serviços no nível coletivo ${ }^{(16)}$ e relacionado com as de- 
mais práticas, conhecimentos, reprodução socioeconomica e inserção dos sujeitos ${ }^{(2)}$, faz-se necessário refletir sobre as potencialidades e os limites do uso de seus instrumentos de trabalho.

O produto CIPESC ${ }^{\circledR}$ Curitiba, de um lado, tem forte direcionamento ao corpo individual e a grupos de patologias ou risco ${ }^{(17)}$, por outro lado, busca romper com a interpretação do processo saúde-doença baseada unicamente na face biológica pois, ao realizar a prática de Enfermagem, contribui captando as necessidades em saúde sob a luz da determinação social do processo saúde-doença. Nessa perspectiva, este estudo apresentou um modelo teórico-informacional de uma ferramenta re-leitora, capaz de construir a ponte necessária entre a dimensão singular e a dimensão particular do processo saúde-doença.

Ao abastecer o data warehouse com dados operacionais, estes são integrados e relacionados de forma a incluir o indivíduo num dos graus de vulnerabilidade propostos, respeitados os parâmetros estabelecidos para a extração dos dados. O prontuário eletrônico do paciente, onde se encontra a base CIPESC $^{\circledR}$, é considerado um banco de dados operacional que contém detalhes do indivíduo, algumas características de sua família e da área em que vive. Desta forma, ao inventariar os dados disponíveis no prontuário eletrônico do paciente, foram construídas subcategorias capazes de se relacionar às categorias interpretativas propostas: necessidade, classe social, gênero e etnia.

Alguns resultados devem ser destacados: na categoria necessidade, os dados estão dispostos de forma a apresentar Necessidades Humanas Básicas; na categoria classe social, o prontuário eletrônico não permite a explicitação de todas as variáveis utilizadas para categorizar classes; na categoria gênero, os dados permitem apenas o acesso à informação relativa ao sexo; a categoria etnia, como identidade de povos, não é explicitada no prontuário eletrônico, nem mesmo por sua possível relação com a variável raça; e a geração faz-se presente como divisão de faixa etária e não na sua dinamicidade com a cultura e modo de vida que determina a construção de identidade.

\section{REFERÊNCIAS}

1. Conselho Internacional de Enfermagem. Classificação Internacional para a Prática de Enfermagem Beta 2. Trad. de Heimar de Fátima Marin. São Paulo; 2003.

2. Pereira MJB, Almeida MCP, Mishima SM, Matumoto S, Pinto $\mathrm{IC}$, Palha PF, et al. O processo de trabalho da enfermagem na rede básica do SUS - Parte II. In: Garcia TR, Nóbrega MML, organizadoras. Sistemas de classificação da prática de enfermagem: um trabalho coletivo. João Pessoa: Idéia/ABEn; 2000. p.7582. (Série Enfermagem no SUS).
Diante deste quadro, bastante limitante, parece impossível o desvelamento da totalidade-parte de uma realidade social. No entanto, algumas potencialidades foram descobertas: os diagnósticos de Enfermagem, construídos na base CIPESC $^{\circledR}$, podem ser agrupados de forma a expressar riscos e contra-valores, bem como potencialidades e valores; as intervenções de Enfermagem, construídas na base CIPESC ${ }^{\circledR}$, podem ser agrupadas nas que superam riscos ou potencializam valores; as frações de classes sociais podem ser hierarquizadas de forma a permitir a organização de diferentes graus de vulnerabilidade dos grupos homogêneos; as categorias Gênero, Etnia e Geração, para serem desveladas, deverão estabelecer ligações com as outras subcategorias.

Uma ferramenta que permite situar o grau de vulnerabilidade de indivíduos pela sua inserção em grupos homogêneos remete à reflexão de que as desigualdades, principalmente étnicas e de gênero, reproduzem-se ao longo do tempo e transformam alguns grupos em alvos fáceis da imobilidade social, historicamente com menor acesso a oportunidades $^{(18)}$. É para estes grupos que se deve oferecer acesso diferenciado a bens e serviços, de forma a propiciar superação da sua condição de vulnerabilidade, na perspectiva da eqüidade, principalmente a vulnerabilidade social, que diz respeito a obtenção de informações, às possibilidades de metabolizá-las e ao poder de incorporar mudanças práti$\operatorname{cas}^{(12)}$. Sendo assim, ao olhar o conjunto de diagnósticos e intervenções de enfermagem, verifica-se que muitas frases listadas situam-se nesta direção, o que nos leva a crer que o trabalho da Enfermagem apreende a vulnerabilidade como uma responsabilidade bilateral e que a CIPESC ${ }^{\circledR}$ tem o potencial para alcançar superações.

Finalmente, com base na Teoria que embasou este estudo, o horizonte que se pretende é dinâmico, processual e histórico $^{(6)}$ e construído à medida que se caminha ${ }^{(6)}$. Portanto, o modelo apresentado carece de operacionalização participativa para validar as relações e hierarquias propostas e possibilitar ampliações ou reduções.

3. Garcia TR, Nóbrega MML. Projeto CIPESC - CIE/ABEn: inventário vocabular de fenômenos e ações de enfermagem em saúde coletiva. In: Garcia TR, Nóbrega MML, organizadoras. Sistemas de classificação da prática de enfermagem: um trabalho coletivo. João Pessoa: Idéia/ ABEN; 2000. p. 83-170. (Série Enfermagem no SUS).

4. Cubas MR, Egry EY. Classificação Internacional de Práticas de Enfermagem em Saúde Coletiva - CIPESC ${ }^{\circledR}$ : revisão de literatura. Rev Esc Enferm USP. 2008;42(1):181-6. 
5. Cubas MR, Lopes MGD, Vaz LA, Albuquerque LM, Perotta SM. Sistematizando a prática da enfermagem na SMS Curitiba. In: Zagonel IPS, Lacerda MR, Lopes MGD, organizadoras. Experiência de enfermeiros da secretaria municipal da saúde de Curitiba: subsídios para sistematização do processo de cuidar em saúde coletiva. Curitiba: ABEn- Seção-PR; 2004. p. 58-64. (Série Enfermagem no SUS).

6. Egry EY. Saúde coletiva: construindo um novo método em enfermagem. São Paulo: Ícone; 1996.

7. Altino DM, Duarte FO, Apostólico MR. Classificação Internacional das Práticas de Enfermagem em Saúde Coletiva-CIPESC: captação da realidade objetiva cenário Curitiba-PR [projeto de pesquisa] São Paulo: Escola de Enfermagem da USP; 2005.

8. Pereira KCM. As práticas de enfermagem em saúde coletiva: conhecendo as ações através da CIPESC [projeto de pesquisa]. São Paulo: Escola de Enfermagem da USP; 2005.

9. Egry EY. Projeto Unificado. In: Egry EY, Cubas MR, organizadoras. O trabalho da enfermagem em saúde coletiva no cenário CIPESC guia para pesquisadores. Curitiba: ABEn-PR; 2006. p. $117-43$.

10. Bursztyn I, Castro VCG, Cohen SC, Ribeiro ALV, Braga MLS, Uribe Rivera FJ. Programa de Saúde da Família: uma análise prospectiva. Cad Saúde Coletiva 2003;11(1):93-112.

11. Inmon WH. Como construir o data warehouse. Trad. de Ana Maria Netto Guz. Rio de Janeiro: Campus; 1997.
12. Ayres JRCM, França Júnior I, Calazans GJ, Saletti Filho HC. O conceito de vulnerabilidade e as práticas de saúde: novas perspectivas e desafios. In: Czeresnia D, Freitas CM, organizadores. Promoção da saúde conceitos, reflexões, tendências. Rio de Janeiro: FIOCRUZ, 2003. p. 117-39.

13. Horta WA. Processo de enfermagem. São Paulo: EPU; 1979.

14. Nascimento ER, Oliva TA. Indicadores de gênero da assistência de enfermagem às mulheres. Rev Bras Enferm. 2004;57(5):565-8

15. Secretaria Municipal da Saúde. Conselho Municipal da Saúde. Relatório da $6^{a}$ Conferência Municipal da Saúde - 2001. Plano Municipal de Saúde de Curitiba: 2002-2005. Curitiba; 2002.

16. Almeida MCP, Rocha SMM. Considerações sobre a enfermagem enquanto trabalho. In: Almeida MCP, Rocha SMM, organizadoras. O trabalho de enfermagem. São Paulo: Cortez; 1997. p. $15-26$.

17. Albuquerque LM, Cubas MR. Cipescar é preciso!...In: Albuquerque LM, Cubas MR, organizadoras. Cipescando em Curitiba: construção e implementação da nomenclatura de diagnósticos e intervenções de enfermagem na rede básica de saúde. Curitiba: ABEn-Seção-PR; 2005. p. 13-7.

18. Heringer R, Miranda D. As cores da desigualdade de gênero no Brasil. In: Araújo C, Scalon C, organizadoras. Gênero, família e trabalho no Brasil. Rio de Janeiro: FGV; 2005. p. 203-24. 